\title{
Determinantes de no-participación en el programa de alimentación complementaria de personas mayores, resultados de la CASEN 2015
}

\section{Determinants of non-participation in the complementary food program for elderly people in Chile, results from the CASEN 2015 survey}

\section{RESUMEN}

Este trabajo se ha centrado en identificar y describir los principales factores sociales que explican el no retiro de alimentos del Programa de Alimentación Complementaria de la Persona mayor (PACAM), según los datos entregados por la encuesta CASEN 2015, dimensión salud. Se usó un modelo lineal no probabilístico, cuyo regresor se refiere a un modelo logit, de respuesta dicotómica, con dos alternativas; la persona mayor no retira los alimentos, la persona mayor si retira los alimentos, del Programa PACAM. El modelo clasifica correctamente el $65,1 \%$ de los casos, con sensibilidad de 59,6\% y especificidad de 70,3\%. Los resultados indicaron que el no retiro de alimentos es preferentemente femenino y se explicó principalmente por el rol de jefe de hogar de la persona mayor, su escasa participación social, habitar en zonas urbanas y cerca de un centro de salud, adicionalmente, la persona mayor presenta problemas de salud, una condición permanente o de larga duración. En conclusión, los resultados indican que las personas mayores que no retiran los alimentos del Programa PACAM, presentan un alto nivel de vulnerabilidad y el tener acceso a dicho Programa impactaría en mejorar su calidad de vida, satisfaciendo sus necesidades nutricionales acorde a su edad. Palabras clave: Calidad de vida; Envejecimiento saludable; Persona mayor; Programa nutricional.

\footnotetext{
ABSTRACT

We focused on identifying and describing the main factors explaining non-withdrawal of food provided by the Complementary Food for Elderly People Program in Chile (PACAM). We used the information contained in the health section of the 2015 Casen survey. Methodologically, we used a binomial probabilistic logit model whose alternatives were whether an elderly person withdraws food (YES/NO) provided by the PACAM program. The model correctly classified $65.1 \%$ of cases with $59.6 \%$ sensitivity and $70.3 \%$ specificity. Results indicated that non-withdrawal occurs mainly by females and related to being the head of household, low social participation, living in urban areas, living near a health center, and the existence a long-term or permanent health problem. In conclusion, main findings show that elderly
}

Pamela Ceroni ${ }^{1}$, Sandra Alvear ${ }^{1}$, Gabriel Pino ${ }^{1}$.

1. Facultad de Economía y Negocios, Universidad de Talca.

Dirigir correspondencia a: Sandra Alvear, Universidad de Talca, Facultad de Economía y Negocios.

Casilla 721 Talca,

Cel.997674752

E-mail: salvear@utalca.cl.

Este trabajo fue recibido el 12 de febrero de 2018. Aceptado con modificaciones: 21 de julio de 2018. Aceptado para ser publicado: 08 de agosto de 2018.

people who do not withdraw food provided by the PACAM program presented a high level of vulnerability. Therefore, access to the PACAM program is important to improve the quality of life by satisfying nutrition needs of elderly people. Keywords: Elderly people; Healthy Aging; Nutrition Programs; Quality of Life.

\section{INTRODUCCIÓN}

El envejecimiento de la población en los últimos años se ha acelerado en todo el mundo, es así como la mayoría de las personas puede aspirar a vivir más allá de los 60 años'. El reto social que este proceso de transición demográfica representa para las naciones se asocia al incremento de las necesidades desde el punto de vista económico, biomédico 
y social. Específicamente, su repercusión sobre el sistema de salud radica en que son las personas mayores los principales consumidores (relativos o absolutos) de medicamentos y servicios de salud ${ }^{2}$.

La sociedad chilena no es una excepción y enfrenta igual desafío, su envejecimiento. Según el último informe de la Encuesta de Caracterización Socioeconómica Nacional (Casen), publicada el año 2015, las personas mayores de 60 años son 3.075.603 habitantes, los que representan el 17,5\% a nivel nacional y el $84 \%$ de ellos es autovalente 3 .

El aumento de la esperanza de vida representa claras oportunidades y desafíos, tanto para la sociedad como para los individuos que conforman este grupo etario, por un lado ellos pueden emprender nuevas actividades y seguir contribuyendo con la sociedad y paralelamente, su estado de salud es un factor que influye en su calidad de vida y que, de alguna forma, condiciona las posibilidades de realizar estas nuevas actividades ${ }^{4}$.

Los cambios psicológicos, sociales y económicos relacionados con el envejecimiento suelen afectar la alimentación de las personas mayores, dicho proceso viene acompañado de cambios fisiológicos que pueden afectar el estado nutricional, las deficiencias sensoriales, en muchos casos disminuyen el apetito y la desnutrición en la vejez a menudo no se diagnostica'. Entre otros factores, es posible advertir una disminución de la capacidad sensorial, relacionada con el olfato, la que se ve acentuada después de los 70 años y representa entonces un problema importante al reducir la apetencia y el placer de comer, también, la sensación subjetiva de sequedad por hiposalivación, la pérdida de piezas dentarias a causa de caries y periodontitis, y el uso de prótesis dentales, las que dificultan la masticación, incidiendo directamente en la disminución del consumo de alimentos de consistencia firme. A nivel del estómago disminuye la secreción de ácido clorhídrico, lo que puede generar una alteración en la absorción de hierro y otros minerales ${ }^{5}$.

Todos estos cambios que las personas mayores experimentan pueden reducirse a través de la modificación de los patrones de alimentación y del estado nutricional, pues los hábitos alimentarios adquiridos durante la infancia y la edad adulta pueden constituirse en un factor protector para la persona mayor, al momento de enfrentar infecciones y enfermedades crónicas asociadas al envejecimiento, incluso pueden ser significativos respecto de la calidad de vida de la que disfrute este grupo etario ${ }^{5}$.

Claramente, este grupo etario se encuentra en una situación de riesgo nutricional, originada por los cambios fisiológicos y/o patológicos que acompañan al proceso de envejecimiento, $y$ por su situación socioeconómica ${ }^{6}$.

En este sentido, los diversos organismos internacionales sugieren a los países realizar esfuerzos que apunten a generar políticas y Programas que promuevan un envejecimiento saludable'. Es así como en Chile se han generado y desarrollado estrategias de intervención específicas en salud, entre otras, el Programa de Alimentación Complementaria de la Persona Mayor (PACAM), a cargo del Ministerio de Salud (MINSAL)․
El Programa PACAM está dirigido a la población beneficiaria de FONASA (Ley № 18.469), con el objetivo de "Contribuir a mantener o mejorar el estado nutricional y la funcionalidad de la persona mayor, aminorando brechas nutricionales y económicas, a fin de mejorar su calidad de vida" ${ }^{\prime \prime}$. Contempla la entrega de alimento instantáneo elaborado en base a cereales, leguminosas o leche, fortificado con vitaminas y minerales. Presentación cuatro variedades: arvejas, lentejas, espárragos, verduras. También, se entrega producto en polvo con base en leche y cereales, fortificada con vitaminas y minerales, reducida en lactosa, baja en grasa total y en sodio. Los principales objetivos de dicho Programa son:

- Entregar un complemento alimentario especialmente diseñado para la persona mayor y acorde a sus necesidades;

- Contribuir a prevenir y tratar las carencias nutricionales de la persona mayor;

- Contribuir a mantener o mejorar las funcionalidad física y psíquica de la persona mayor;

- Contribuir con la detección y control de los factores de riesgo de pérdida de funcionalidad física y psíquica de la persona mayor;

- Incentivar una mayor adherencia a las actividades del Programa del Salud de la Persona Mayor en Aporte Previsional Solidario (APS);

- Promover en los establecimientos de salud y postas rurales, una modalidad de trabajo integral con relación a la persona mayor, con participación de profesionales del área de atención médica, nutricional, asistencia social, de la familia y de las organizaciones de la propia comunidad.

En un estudio realizado el año 2008, se determinó que el Programa PACAM alcanzaba una cobertura del $40 \%$ de las personas mayores de 70 años y más, beneficiarios de Fonasa, incrementándose en tres puntos respecto al período 2007, el mismo estudio plantea que el gran desafío de los Programas alimentarios es su actualización, siendo prioritario avanzar en la evaluación y análisis del impacto económico y nutricional de estos Programas ${ }^{8,9}$.

Por otra parte, según datos de la encuesta CASEN 2015, el $48,1 \%$ de la población beneficiaria de FONASA no retira alimentos del PACAM $^{3}$ y dicho Programa representa un porcentaje importante del presupuesto asignado a las acciones de salud en el nivel primario ${ }^{10}$. Sin embargo, se desconocen las características y determinantes de dicho grupo, así como las motivaciones que tienen para el no retiro; considerando que se está frente a un Programa diseñado especialmente para las personas mayores, los recursos asociados a dicho Programa y el impacto que debería tener en dicho grupo etario, conforman los fundamentos básicos que motivan la realización de este estudio.

Por esta razón, tratándose del impacto en la calidad de vida de las personas mayores y por otra parte, de la gran inversión de recursos que representa este Programa para el país, es que se hace necesaria la identificación de las determinantes de la población beneficiaria del Programa de alimentación complementaria que no hace uso de este beneficio. En atención 
a lo antes expuesto, este trabajo pretende "identificar las principales características de las personas mayores en Chile que no retiran los alimentos del Programa de Alimentación Complementaria de la Persona Mayor (PACAM), según CASEN 2015" con la finalidad de contribuir a la gestión de dicho Programa y, por ende, al mejoramiento de la calidad de vida y bienestar de este grupo etario.

\section{MATERIAL Y MÉTODOS}

Se trata de un análisis de datos secundarios, a partir de Encuesta de Caracterización Socioeconómica Nacional (Casen), cuyos resultados se ponen a disposición del público, a través de una base de datos, disponible en el Instituto Nacional de Estadística (INE). Dicha encuesta es de hogares, de carácter transversal y de multipropósitos. La encuesta Casen, metodológicamente, en el análisis de los datos propone dos alternativas de factor de expansión. En el presente estudio se usó el expansor regional, que extiende la proyección de la población regional ${ }^{3}$.

A partir de los datos obtenidos de la Casen 2015, se realizó un estudio exploratorio correlacional. El universo de este estudio es el grupo de la población de 70 años y más, registrados en el Fondo Nacional Salud (FONASA), según la definición de beneficiario que establece el Programa de Alimentación Complementaria de la Persona Mayor (PACAM). Cabe destacar que según la Ley $N^{\circ} 19.828$ del año 2015, se fija la adultez mayor en 60 años $^{11}$. La unidad de análisis son los beneficiarios del Programa que no retiran alimentos de los establecimientos de salud primaria.

Los datos de las personas mayores se encuentran anonimizados en la encuesta, por ello no fue necesario solicitar consentimiento informado ${ }^{12}$.

No se consideró en el estudio los beneficiarios del Programa de Reparación y Atención Integral de Salud, PRAIS (Ord. B34/3560 del 30 de octubre, 2013), pertenecientes a ISAPRE, personas mayores de 65 años con tratamiento antituberculoso, beneficiarios del Programa Chile Solidario y del Hogar de Cristo, dado que la encuesta Casen, no permite identificar estos grupos.

La variable dependiente del estudio es "personas beneficiarias del Programa PACAM", obtenida, a partir de la respuesta dada a la siguiente pregunta: En los últimos tres meses, ¿recibió o retiró, gratuitamente, alimentos del consultorio? Para el análisis las respuesta se agruparon en dos categorías: No, retiro alimentos = 1 y Sí, retiro alimentos (bebidas lácteas y cremas de años dorados $)=0$.

Como variables independientes se definió: Género, con valor 1 si se es hombre y 0 si se es mujer. Residencia, con valor 1 si reside en sector urbano y 0 si reside es sector urbano. Jefe de Hogar, con valor 1 si se es jefe de hogar y 0 si no se es. Pareja, toma el valor 1 si la persona es casado, conviviente o pareja sin o con acuerdo de unión civil y 0 si es anulado, separado, divorciado, viudo o soltero. Control de salud, con valor 1 si la persona realizó un control de enfermedades crónicas, preventivo de la persona mayor, dental o de otro tipo y 0 si no efectúo control alguno. Problemas de salud, con valor 1, si la persona en los últimos tres meses presentó un problema de salud, enfermedad o accidente, y 0 si no presentó problemas de salud. Condición permanente, toma el valor 1 , si la persona tiene dificultad física y/o movilidad, mudez o dificultad del habla, dificultad psiquiátrica, dificultad mental o intelectual, sordera o dificultad para oír, ceguera o dificultad para ver y toma el valor 0 , si no presenta dicha condición. Distancia de un centro de salud, toma el valor 1 si la persona vive a 2,5 km. de un centro de salud (APS o nivel superior) y 0 si la persona vive a mayor distancia. Participación social, toma valor $1 \mathrm{si}$ la persona participa de alguna agrupación social y 0 si no participa. Quintil de Ingreso, variable dividida en cinco sub variables (Quintil de Ingresos 1, 2, 3, 4 y 5), cada una de ellas toma el valor 1 , si la persona pertenece al tramo de ingresos y 0 sino pertenece. Región, dividida en las 15 regiones del país, tomando el valor 1 si la persona habita en la región y 0 sino habita en ella. Tramo de Salud FONASA, dividida en los tramos A, B, C y D, tomando el valor 1 si la persona pertenece a un tramo y 0 si no pertenece a él. Tramo de edad, dividida en dos tramos (70-79; 80 y más), tomando el valor 1 si la persona tiene una edad perteneciente a un tramo y 0 si no pertenece a él. Escolaridad, dividida en las categorías básica, media, superior y sin escolaridad, tomando el valor 1 si tiene escolaridad, expresada en años de estudios, y 0 si no la tiene.

\section{Análisis estadístico}

El análisis estadístico de los datos se realizó con el apoyo del Software Stata ${ }^{13}$. Se usó un modelo no lineal probabilístico, con dos alternativas de respuesta: la persona mayor no retira los alimentos del PACAM y, la persona mayor sí retira los alimentos del PACAM. El regreso se refiere a un modelo logit. La función utilizada es logística, en donde se plantea la hipótesis de distribución binomial de la variable respuesta13 y la especificación del modelo es:

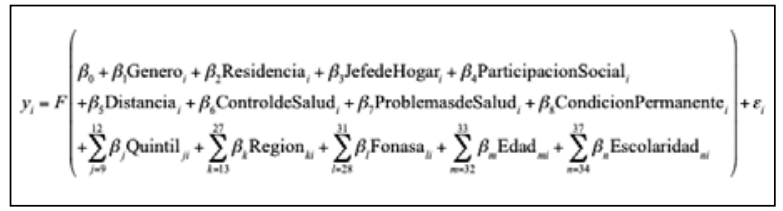

Donde $F($.$) corresponde la función de distribución$ acumulada que se asume logística la cual cumple el rol de enlace entre los determinantes y la probabilidad de retirar el alimento del programa PACAM. Por lo tanto, el modelo logit a estimar corresponde a:

$$
y_{i}=\frac{e^{\sum_{p-0}^{34} \beta_{p} X_{p i}}}{1+e^{\sum_{p=0}^{34} \beta_{p} X_{p i}}}+\varepsilon_{i}
$$

Donde Xpi corresponde a los 37 determinantes previamente especificados. 
Los estadísticos de bondad de ajuste en regresión logística usados son: Test de McFadden's, Test de Cox y Snell, Test de Nagelkerke y los estadísticos Akaike Information Criteria (AIC).

La encuesta CASEN 2015 está diseñada para ser representativa a nivel nacional, regional, por zona de residencia (urbana y rural), y en comunas que, sumadas, concentran el $80 \%$ ó más de las viviendas de cada región (según marco muestral INE) ${ }^{3}$. De total de la encuestas Casen 2015, para efectos del presente estudio, se seleccionó aquellas cuyos Jefes de Hogar eran mayores 70 años, definidas como personas mayores beneficiarios del Programa PACAM, de acuerdo a su afiliación a FONASA, conformando así una muestra de 1.234.118 personas.

\section{RESULTADOS \\ Características de la población}

Según la encuesta CASEN 2015, el 51,9\% de las personas mayores retiran los alimentos disponibles, en el contexto de dicho Programa y el $48,1 \%$ no los retira, siendo beneficiaria. Las personas mayores que no retiran los alimentos del Programa PACAM, son mayoritariamente mujeres, habitan en zonas urbanas, a menos de 20 cuadras de un centro de salud (cerca), no poseen control médico, presentan problemas de salud, enfermedad o accidente y poseen una condición permanente o de larga duración. Todas estas características, con excepción de vivir en zona urbana, son estadísticamente significativas al comparar con proporciones de situación con PACAM. Las demás características se presentan en la Tabla 1, la cual también presenta el estadístico chi-cuadrado para la hipótesis nula de igualdad de proporciones en el caso con PACAM y sin PACAM.

\section{Bondad de Ajuste del Modelo}

En la predicción del retiro de alimentos del Programa PACAM, en 3 iteraciones se ha logrado estimar los coeficientes que más verosímilmente pueden haber producido los valores observados de la variable dependiente, beneficiarios del Programa PACAM (Tabla 2).

Según la prueba estadística basada en $x^{2}$ el modelo es significativo con un $95 \%$ de confianza, es decir, la relación entre los coeficientes del modelo y la probabilidad de que una persona mayor no retire los alimentos del Programa PACAM es estadísticamente significativa (Tabla 2).

El test $L R^{33}$, prueba de verosimilitud, muestra la diferencia entre el modelo sólo con la constante y el modelo completo, y según Prob $>L R$, se rechaza la hipótesis nula, es decir, al menos uno de los coeficientes que aparecen en el modelo logístico estimado es significativamente distinto de cero. El valor del estadístico McFadden's R2 está más próximo a cero que a uno (Tabla 2).

Según el estadístico Count R2 el modelo logit acierta en un $65,1 \%$, este valor corregido según el estadístico Adj Count R2. El modelo estimado incrementa la capacidad de acierto en un $27,6 \%$, con respecto a la que se tendría simplemente prediciendo que las personas mayores retiran los alimentos, es decir, el resultado más común, y esto indicaría que el

Tabla 1. Características de la población de Personas Mayores Fuente: Elaboración propia a partir de los datos del estudio. a Hipótesis nula de igualdad de proporciones con PACAM vs sin PACAM es rechazada al $1 \%$ de significancia.

\begin{tabular}{|c|c|c|c|c|c|c|}
\hline Género & $\begin{array}{l}\text { Mujer } \\
\text { Hombre } \\
\text { Total }\end{array}$ & $\begin{array}{l}397.254 \\
242.754 \\
640.008\end{array}$ & $\begin{array}{c}62 \% \\
38 \% \\
100 \%\end{array}$ & $\begin{array}{l}343.652 \\
250.458 \\
594.110\end{array}$ & $\begin{array}{c}58 \% \\
42 \% \\
100 \%\end{array}$ & $\begin{array}{c}\text { Estadístico } \\
296.27^{\mathrm{a}} \\
21.43^{\mathrm{a}}\end{array}$ \\
\hline Residencia & $\begin{array}{l}\text { Urbana } \\
\text { Rural }\end{array}$ & $\begin{array}{l}506.482 \\
133.526\end{array}$ & $\begin{array}{l}79 \% \\
21 \%\end{array}$ & $\begin{array}{c}526.143 \\
67.967\end{array}$ & $\begin{array}{l}89 \% \\
11 \%\end{array}$ & $\begin{array}{c}0.62 \\
1042.48^{\mathrm{a}}\end{array}$ \\
\hline $\begin{array}{l}\text { Distancia Centro } \\
\text { Salud }\end{array}$ & $\begin{array}{l}\text { Cerca (menos de } 20 \text { cuadras) } \\
\text { Lejos (más de } 20 \text { cuadras) }\end{array}$ & $\begin{array}{c}524.891 \\
115.117\end{array}$ & $\begin{array}{l}82 \% \\
18 \%\end{array}$ & $\begin{array}{c}502.484 \\
91.626\end{array}$ & $\begin{array}{l}85 \% \\
15 \%\end{array}$ & $\begin{array}{l}101.93^{\mathrm{a}} \\
230.84^{\mathrm{a}}\end{array}$ \\
\hline $\begin{array}{l}\text { Control } \\
\text { Salud }\end{array}$ & $\begin{array}{l}\text { Con Control } \\
\text { Sin Control }\end{array}$ & $\begin{array}{l}450.122 \\
189.886\end{array}$ & $\begin{array}{l}70 \% \\
30 \%\end{array}$ & $\begin{array}{l}265.233 \\
328.877\end{array}$ & $\begin{array}{l}45 \% \\
55 \%\end{array}$ & $\begin{array}{c}2190.42^{\mathrm{a}} \\
972.15^{\mathrm{a}}\end{array}$ \\
\hline $\begin{array}{l}\text { Condición } \\
\text { permanente/larga } \\
\text { duración }\end{array}$ & $\begin{array}{l}\text { Con } \\
\text { Sin }\end{array}$ & $\begin{array}{l}253.790 \\
386.218\end{array}$ & $\begin{array}{l}40 \% \\
60 \%\end{array}$ & $\begin{array}{l}190.195 \\
403.915\end{array}$ & $\begin{array}{l}32 \% \\
68 \%\end{array}$ & $\begin{array}{c}17.65^{\mathrm{a}} \\
465.22^{\mathrm{a}}\end{array}$ \\
\hline
\end{tabular}


modelo tienen cierta capacidad para predecir el retiro de los alimentos de las personas mayores (Tabla 2).

En general, el modelo clasifica correctamente el $65,13 \%$ de los casos y explica entre $12,9 \%$ y el $17,3 \%$ de los casos. Según el índice de sensibilidad, del total de personas mayores beneficiarias del Programa PACAM, el modelo clasificó correctamente el 59,57\% de los casos que no retiran los alimentos y de acuerdo con el índice de especificidad, el modelo clasificó el 70,29\% de los casos que sí retiran alimentos (Tabla 2).

Tabla 2. Estadísticos de bondad de ajuste para modelos logísticos binarios

$\begin{array}{lc}\text { Número de Observaciones } & 1.234 .118 \\ \text { Iteraciones } & (3)-768716.57 \\ \text { LR(33) } & 171710.2 \\ \text { Prob > LR } & 0,000 \\ \text { McFadden's Adj R2 } & 0,1 \\ \text { Count R2 } & 65,1 \% \\ \text { Adj Count R2 } & 27,6 \% \\ \text { AIC } & 1,246 \\ \text { Correctamente clasificado } & 65,13 \% \\ \text { Cox-Snell } & 0,129 \\ \text { Nagelkerke } & 0,173 \\ \text { Sensibilidad } & 59,57 \% \\ \text { Especificidad } & 70,29 \% \\ \text { Constante } & 1,505\end{array}$

Fuente: Elaboración propia a partir de los datos del modelo

\section{Estimaciones del modelo}

Según los valores estimados para los cocientes de razones (OR), Tabla 3, las variables que tienen mayor impacto en la estimación del uso del Programa PACAM son: género, jefe de hogar, participación social, distancia de un centro de salud, problemas de salud, condición permanente, tramo de edad y escolaridad. Para cada una de las variables, los intervalos de confianza al 95\% no contienen el valor unitario (1).

Considerando el quintil de ingreso per cápita al que pertenece el beneficiario del PACAM se puede destacar que en comparación con el quintil 5 todos los otros quintiles disminuyen la razón de no retiro de alimentos.

La razón de no retiro de alimentos, según la región del país en la que resida la persona mayor, aumenta con respecto de la Región Metropolitana de Santiago, si la persona mayor reside en la Región de Tarapacá, Antofagasta, Atacama, Aysén y Magallanes.

Otro variable considerada en este estudio dice relación con el tramo de FONASA al que se encuentra asimilado un persona mayor, es así como se advierte que si un persona mayor se encuentra asimilado al tramo A, B o C disminuye la razón de no retiro de alimentos PACAM con respecto a aquel perteneciente al tramo $\mathrm{D}$.

Si se considera el rango etario, si una persona mayor tiene entre 70 y 79 años de edad aumenta la razón de no retiro de alimentos, respecto de una persona mayor que tiene más de 80 años de edad.

Finalmente, si se consideran los años de escolaridad, se observa, con respecto a una persona mayor sin escolaridad, que la razón de no retiro aumenta en la medida que aumentan los años de escolaridad.

\section{DISCUSIÓN}

El envejecimiento de la población chilena es un tema relevante desde la perspectiva de la salud pública'. En la misma línea existen estudios que muestran que una buena nutrición y un estilo de vida activo, influyen positivamente en la autopercepción de salud de las personas mayores ${ }^{14}$. Una alimentación o nutrición adecuada de acuerdo a la edad de la persona mayor es un factor relevante en la calidad de vida de éstos ${ }^{15}$. El envejecimiento viene acompañado de cambios fisiológicos que pueden afectar el estado nutricional, las deficiencias sensoriales en muchos casos disminuyen el apetito y la desnutrición en la vejez a menudo no se diagnostica, y son pocas las evaluaciones exhaustivas de la prevalencia mundial de las diferentes formas de desnutrición ${ }^{1,16}$.

Sin embargo, en la persona mayor chilena el problema fundamental no parece estar en el enflaquecimiento, sino en una alimentación deficiente en micronutrientes, que afecta negativamente diversas funciones del organismo ${ }^{17}$. En tanto, es importante tener presente que según el presente estudio la razón de no retiro de alimentos del Programa PACAM aumenta, con respecto a una persona mayor sana. Se puede inferir que dicha situación obedece a la escasez de redes de apoyo que faciliten el acceso de las personas mayores a los centros de salud en donde se entregan dichos alimentos.

Los resultados del presente estudio indican que el no retiro de alimentos del Programa PACAM es preferentemente femenino, lo anterior en línea con una sociedad que presenta un envejecimiento muy heterogéneo y mayoritariamente femenino ${ }^{3}$.

En un estudio realizado en Colombia sobre hábitos alimentarios de las personas mayores, desde el punto de vista cualitativo, se destaca que a su edad se limita el acceso a los alimentos, entre otros por depender de otras personas para el acceso a los alimentos, en tanto, sus hábitos alimentarios contribuyen a su proceso de mayor fragilidad ${ }^{5}$. En la misma línea, el presente estudio muestra que si una persona mayor no participa de actividades sociales, se incrementa la razón de no retiro de alimentos del Programa PACAM, con respecto a una persona mayor que sí participa. De ahí la importancia de las redes de apoyo con las que deberían contar dicho grupo etario.

Si una persona mayor tiene una condición permanente, tal como limitación física, ceguera o dificultad para ver, sordera o dificultad para oír, u otra condición, la razón de no retiro de alimentos aumenta, con respecto a una persona 
Tabla 3. Estimación de determinantes de la población que No retira alimentos PACAM

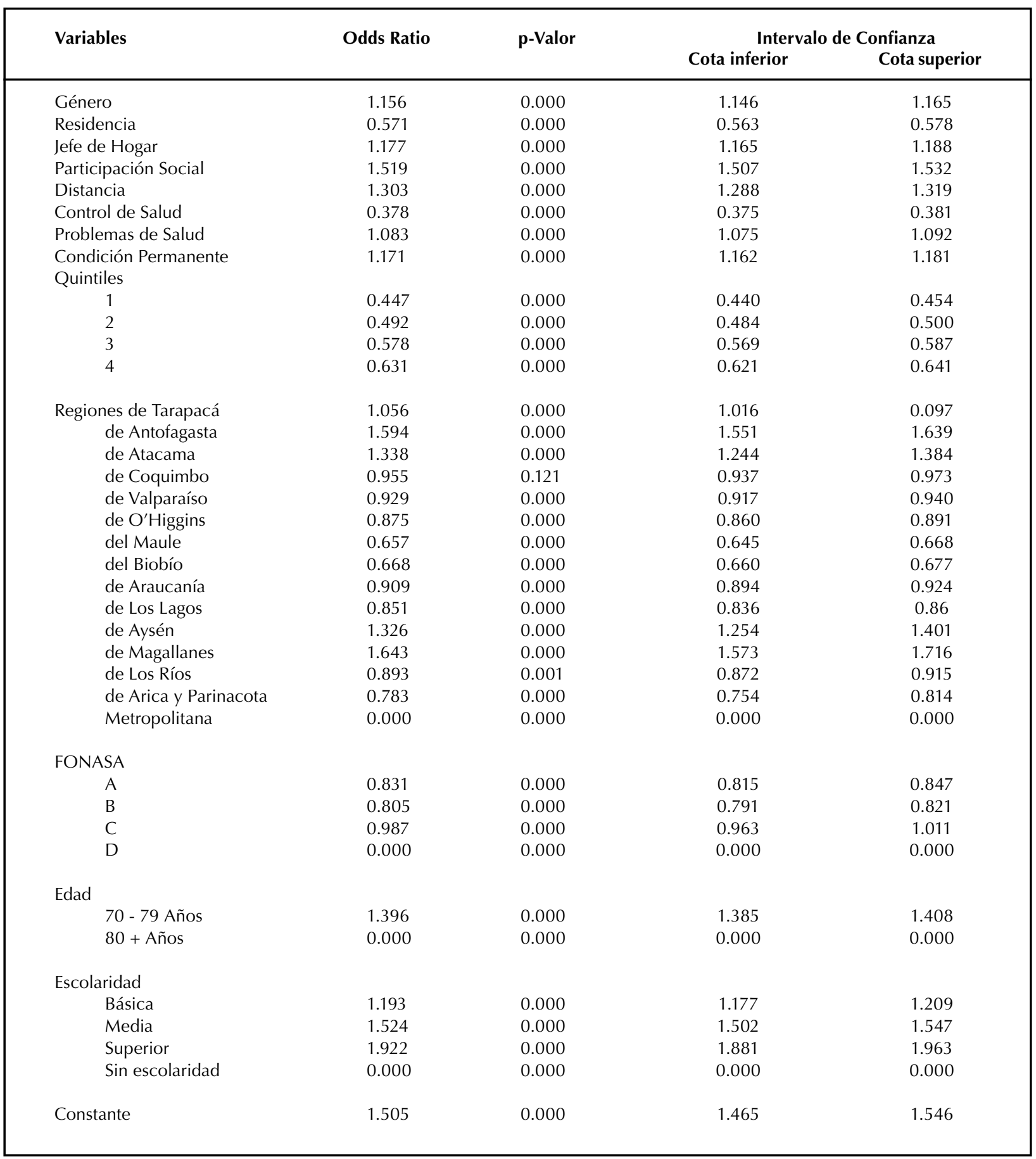

mayor que no presenta tal condición. En la misma línea, existe un estudio que muestran que el deterioro gradual de las condiciones de salud física y mental que acompañan el envejecimiento individual, con el consecuente aumento de problemas de salud crónicos, conducen -de no mediar intervenciones específicas- a limitación funcional y gradual pérdida de autonomía ${ }^{17}$.

Finalmente, es importante destacar que el hecho de que 
un $48,1 \%$ de las personas mayores no reciban los alimentos del Programa PACAM, sin duda, tiene un impacto económico en el sector salud, dado que dicho Programa, para el año 2017 en miles de \$ representaba 23.963.315, equivalente al 5,52\% del presupuesto asignado a la Subsecretaria de Salud Pública ${ }^{10}$.

\section{CONCLUSIONES}

Según datos CASEN 2015, el 48,1\% de las personas mayores, pertenecientes a FONASA, no retira alimentos fortificados con micronutrientes ofrecidos a través del Programa PACAM, impactando negativamente en dos dimensiones, en primer lugar, en el estado nutricional de las personas mayores vulnerables y en su calidad de vida y en segundo lugar, en el costo alternativo de los recursos destinados a adquirir dichos productos, en un contexto de recursos limitados. Es necesario continuar realizando evaluaciones del impacto social de dicho Programa, generando nuevas modalidades de distribución de los alimentos, con el objetivo de hacerlos accesible a las personas mayores vulnerables que hoy no están accediendo a ellos.

Investigación, financiada por CONICYT mediante el proyecto FONDEF ID16AM0006: El maltrato estructural a las personas mayores en Chile. Formulación de un índice multidimensional (2017-2019).

\section{BIBLIOGRAFÍA}

1. Organización Mundial de la Salud. (2015). Informe Mundial sobre el Envejecimiento y la Salud. Ginebra: Ediciones de la OMS. Acceso: 14/11/2017. Disponible en: http://www.afro. who.int/sites/default/files/2017-06/9789240694873_spa.pdf.

2. Alonso P, Sansó FJ, Díaz-Canel AM, Carrasco M, Oliva T. Envejecimiento poblacional y fragilidad en el persona mayor. Rev Cub Salud Pública 2007; 33(1): 1-18.

3. Encuesta de Caracterización Socioeconómica Nacional (CASEN). 2015. Acceso: 14/11/2017. Disponible en:http://observatorio. ministeriodesarrollosocial.gob.cl/casen-multidimensional/ casen/casen_2015.php

4. Castañeda IE, Nelson ON. Necesidades de servicios de salud en mujeres y hombres mayores de 60 años. Rev Cubana Med Gen Integr 2013; 29 (1): 36-43.

5. Lutz M, Morales D, Sepúlveda S, Alviña M. Evaluación sensorial de preparaciones elaboradas con nuevos alimentos funcionales destinados al persona mayor. Rev Chil Nutr 2008; 35(2): 131-137.

6. Restrepo SL, Morales RM, Ramírez MC, López MV, Varela LE. Los hábitos alimentarios en el persona mayor y su relación con los procesos protectores y deteriorantes en salud. Rev Chil Nutr 2006; 33(3): 500-510.

7. Ministerio de Salud. Programa de Alimentación Complementaria del Persona mayor (PACAM). Acceso: 14/11/2017. Disponible en http://minsal.cl/departamentos-2/nutricion-y-alimentos/ Programas-alimentarios/pacam/

8. Barba C, Jiménez M, Pizarro T, Rodríguez L. Protección social en los Programas alimentarios del Ministerio de Salud de Chile. Cuad Méd Soc (Chile) 2008; 48(4): 240-250.

9. Riumalló J, Pizarro T, Rodríguez L, Benavides X. Programas de suplementación alimentaria y de fortificación de alimentos con micronutrientes en Chile. Cuad Méd Soc (Chile) 2009; 43(1): 53-60.

10. Ley de Presupuesto del sector público año 2017. Acceso: 14/11/2017. Disponible en http://dipres.gob.cl/598/ articles-149470_Ley_de_Presupuestos_2017_V2.pdf

11. Ley $N^{\circ} 19.828 ;$ Convención Interamericana sobre Protección de Derechos de Personas Mayores, 2011. Acceso: 14/11/217. Disponible en: Biblioteca del Congreso Nacional. https:// www.leychile.cl/Navegar?idNorma=202950.

12. Peña C. (2012). Ëtica y derecho en la investigación en ciencias sciales en Bioética en Investigación en Ciencias Sociales. Acceso: 14/11/2017. Disponible en: http://www. conicyt.cl/fondecyt/files/2012/10/Libro-3-Bio\%C3\%A9ticaen-investigaci\%C3\%B3n-en-ciencias-sociales.pdf.

13. Collett D. Modelling Binary Data. Chapman and Hall. London, 1991.

14. Jewell T, Rossi M, Triunfo P. El estado de salud del Persona mayor en América Latina. Cuad Econ 2007; 26(46): 147-167.

15. Osorio P, Torrejón MJ, Anigstein MS. Calidad de vida en personas mayores en Chile. Revista Mad-Universidad de Chile 2011; 24: 61-75.

16. Reyes I, Castillo JA. El envejecimiento humano activo y saludable, un reto para el anciano, la familia, la sociedad. Rev Cubana Invest Bioméd 2011; 30(3): 354-359.

17. Urteaga C, Ramos RI, Atalah E. Validación del criterio de evaluación nutricional global del persona mayor. Rev med Chile 2001; 129(8): 871-876.

18. Albala C, Sánchez H, Lera L, Angel B, Cea X. Efecto sobre la salud de las desigualdades socioeconómicas en el persona mayor. Resultados basales del estudio expectativa de vida saludable y discapacidad relacionada con la obesidad. Rev med Chile 2011; 139(10): 1276-1285. 